\title{
A NOTE ON SPECIAL KÄHLER MANIFOLDS
}

\author{
ZHIQIN LU
}

The base space of an algebraically completely integrable Hamiltonian system acquires a rather special differential-geometric structure which plays an important role in modern physical theories such as SeibergWitten theory. This structure was formalised by D. Freed [1] as a Special Kähler manifold. In that paper he conjectured that there are no compact special Kähler manifolds other than flat ones. In this paper, we prove that there are no nonflat complete special Kähler manifolds, thus verifying the conjecture.

Definition 1. Let $M$ be a Kähler manifold with Kähler form $\omega$. A special Kähler structure on $M$ is a real flat torsionfree symplectic connection $\nabla$ satisfying

$$
d_{\nabla} I=0
$$

where $I \in \Omega^{1}(M, T M)$ is the complex structure on $M$.

The following property is true for any special Kähler manifold.

Theorem 1. Any special Kähler manifold has nonnegative Ricci curvature. Moreover, if the scalar curvature is identically zero, then the curvature tensor itself vanishes.

Proof: We take $\left(z^{1}, \cdots, z^{n}\right)$ to be a special coordinate system. Under this coordinate system, the Kähler form can be represented as

$$
\omega=\frac{\sqrt{-1}}{2} h_{i \bar{j}} d z^{i} \wedge d \bar{z}^{j}=\frac{\sqrt{-1}}{2} \operatorname{Im} u_{i j} d z^{i} \wedge d \bar{z}^{j}
$$

where $u$ is a local holomorphic function [1, page 5] and $u_{i j}$ is defined as $\frac{\partial^{2} u}{\partial z^{i} \partial z^{j}}$. Moreover, we define $u_{i_{1} \cdots i_{n}}$ to be $\frac{\partial^{n} u}{\partial z^{1} \ldots \partial z^{n}}$.

It is then a straightforward computation that

$$
R_{i \bar{j} k \bar{l}}=-\frac{1}{4} h^{m \bar{n}} u_{i k m} \overline{u_{j l n}}
$$

Date: July 3, 2018.

1991 Mathematics Subject Classification. Primary: 58G03; Secondary: 35J05.

Key words and phrases. special Kähler manifolds, generalised maximal principle, curvature tensor. 
where our definition for the curvature tensor is

$$
R_{i \bar{j} k \bar{l}}=\frac{\partial^{2} h_{i \bar{j}}}{\partial z^{k} \partial \bar{z}^{l}}-h^{m \bar{n}} \frac{\partial h_{m \bar{j}}}{\partial \bar{z}^{l}} \frac{\partial h_{i \bar{n}}}{\partial z^{k}}
$$

Let $F$ be the cubic form defined as

$$
F=-\omega\left(\pi^{(1,0)}, \nabla \pi^{(1,0)}\right)
$$

where $\pi^{(1,0)} \in \Omega^{1,0}\left(T_{C} M\right)$ is the projection onto the $(1,0)$ part of the complexified tangent bundle. $F$ is a global section of the bundle $S y m^{3} T^{*} M$. Locally $F_{i j k}=u_{i j k}$. Thus we have

$$
R_{i \bar{j} k \bar{l}}=-\frac{1}{4} h^{m \bar{n}} F_{i k m} \overline{F_{j l n}}
$$

We remark that the above identity is valid for any local holomorphic coordinate system, because both sides are tensors. From (11) we see that the Ricci curvature is nonnegative. Furthermore, if the scalar curvature $\rho$

$$
\rho=\frac{1}{4} h^{i \bar{i}_{1}} h^{j \bar{j}_{1}} h^{k \bar{k}_{1}} F_{i j k} \overline{F_{i_{1} j_{1} k_{1}}}
$$

is identically zero, then the curvature tensor vanishes.

Theorem 2. If $(M, \omega)$ is a complete special Kähler manifold, then the curvature tensor vanishes.

Proof: Define

$$
F_{i j k, l}=\partial_{l} F_{i j k}-\Gamma_{i l}^{m} F_{m j k}-\Gamma_{j l}^{m} F_{i m k}-\Gamma_{k l}^{m} F_{i j m}
$$

to be the covariant derivative of $F_{i j k}$. By the Bochner formula

$$
\begin{aligned}
& \Delta \rho=\frac{1}{4} h^{i \bar{i}_{1}} h^{j \bar{j}_{1}} h^{k \bar{k}_{1}} h^{l \bar{l}_{1}} F_{i j k, l} \overline{F_{i_{1} j_{1} k_{1}, l_{1}}} \\
& +\frac{3}{4} h^{m_{1} \bar{i}_{1}} h^{i \bar{m}} h^{j \bar{j}_{1}} h^{k \bar{k}_{1}} R_{m_{1} \bar{m}} F_{i j k} \overline{F_{i_{1} j_{1} k_{1}}}
\end{aligned}
$$

where $R_{m_{1} \bar{m}}=-h^{\alpha \bar{\beta}} R_{\alpha \bar{\beta} m_{1} \bar{m}}$ is the Ricci tensor. In particular, we have

$$
\Delta \rho \geq 3 h^{m_{1} \bar{i}_{1}} h^{i \bar{m}} R_{i \bar{i}_{1}} R_{m_{1} \bar{m}} \geq \frac{3}{n} \rho^{2}
$$

We use the version of the generalized maximal principle in 2, page 582, Lemma 1.1]. The original statement in [2] is quite general. We rewrite it in the following (simplified) way: 
Proposition 1. Let $M$ be a complete Kähler manifold of nonnegative Ricci curvature. Let $\varphi$ be a nonnegative function satisfying

$$
\Delta \varphi \geq C_{1} \varphi^{\alpha}-C_{2} \varphi-C_{3}
$$

where $\alpha>1, C_{1}>0, C_{2}, C_{3} \geq 0$ are constants. Then

$$
\sup \varphi \leq \operatorname{Max}\left\{1,\left(\frac{C_{2}+C_{3}}{C_{1}}\right)^{1 / \alpha}\right\}
$$

For any positive number $a$, from (44) we see that $\Delta(a \rho) \geq \frac{3}{n a}(a \rho)^{2}$. By the above proposition we see that $a \rho \leq 1$. Letting $a \rightarrow \infty$ we see that $\rho \equiv 0$. The theorem thus follows from Theorem [1.

Acknowledgment. The author thanks Professor G. Tian for the support during the preparation of this paper. He thanks Professor D. Freed for some very useful conversations about this topic, from which he learned a lot about the special Kähler geometry. He also thanks Professor N. Hitchin for the interest of the paper and suggestions which make the paper in its current form.

\section{REFERENCES}

[1] Daniel S. Freed. Special Kähler Manifolds. hep-th/9712042.

[2] Gang Tian and Shing-Tung Yau. Existence of Kähler-Einstein Metrics on Complete Manifolds and their Applications to Algebraic Geometry. In Shing-Tung Yau, editor, Mathematical aspects of string theory, volume 1, pages 574-628. World Scientific, 1987.

(Zhiqin Lu) Department of Mathematics, Columbia University, NY, NY 10027

E-mail address: lu@cpw.math.columbia.edu 